\section{FLUID FREE BILAYERS: A MODEL SYSTEM TO STUDY} MEMBRANES

$\underline{\text { T. Charitat }}^{1}$ E. Bellet-Amalric ${ }^{2}$ A. Braslau ${ }^{3}$ J. Daillant ${ }^{4}$ G. Fragneto ${ }^{5}$ F. Graner $^{6}$ K. Mecke

${ }^{1}$ Institut Charles Sadron 6 Rue Boussingault Strasbourg 67083 France ${ }^{2}$ DRFMC-Sp2m/Sgx Cea Grenoble ${ }^{3}$ Cea, Saclay ${ }^{4}$ Lure, Orsay ${ }^{5}$ Ill-Grenoble

${ }^{6}$ Lsp-Grenoble ${ }^{7}$ Bergische Universitat Wuppertal

Physical and biophysical studies of membrane-membrane and membraneprotein interactions require well controlled model systems. We have developed a new method to obtain a single lipid bilayer floating in excess of water, in the vicinity of a fixed bilayer. It has been prepared in the gel phase, which is not biophysically relevant.

The free bilayer has been precisely characterized by Neutron and X-Ray reflectivity. By increasing the temperature up to the melting transition of the lipid chains, one can observe a spectacular change in bilayer-bilayer distance. We attribute this swelling to the bilayer's interaction of Helfrich due to the bilayer fluctuations. Theoretical implications will be discussed, allowing us to measure the bending modulus of the membrane in a large range of temperatures. In order to characterize more precisely the fluctuations of the membrane we have developed X-Ray off-specular reflectivity leading to a direct measurement of the fluctuations spectrum of the membrane.

As a conclusion we have obtained a free lipid bilayer, fluid and stable, biophysically relevant substrate for bilayer-bilayer or protein-bilayer interaction studies. This allows us to characterize the fluctuations spectrum of liquid membrane in a large range of temperatures.

\section{Keywords: LIPID BILAYER FLUCTUATIONS REFLECTOMETRY}

Acta Cryst. (2002). A58 (Supplement), C220

HOMOCHIRAL OLIGOPEPTIDES OBTAINED BY LATTICE-CONTROLLED REACTIONS IN TWO-DIMENSIONAL CRYSTALLINE ASSEMBLIES ON WATER

I. Weissbuch ${ }^{1}$ K. Kjaer ${ }^{2}$ L. Leiserowitz ${ }^{1}$ M. Lahav ${ }^{1}$

${ }^{1}$ Department of Materials and Interfaces, Weizmann Institute of Science, 76100-Rehovot, Israel ${ }^{2}$ Materials Research Department, Risoe National Laboratory, 4000-Roskilde, Denmark

Enantioselective reactions starting from heterochiral mixtures of precursors might have been involved during the formation of homochiral biopolymers of life at prebiotic times. Since reactions in isotropic media would lead to heterochiral products, a way to obtain oligopeptides of homochiral sequences from racemic reactants would be through the assembly of the precursor molecules into ordered architectures followed by latticecontrolled reactions. Two-dimensional (2-D) self-assemblies of amphiphilic molecules formed on water surface provide an ideal medium. Racemates of reactive $\alpha$-amino acid amphiphiles were designed to self-assemble, on water surface, into 2-D crystallites of three types: racemic compounds, enantiomorphous conglomerates and enantiomerically disordered solid-solutions. Such self-assemblies could undergo polymerization to yield mixtures of oligopeptides of enantiomeric composition controlled by the packing motif of the precursor crystalline phase.

The 2-D self-assembly of various racemates and the reaction induced by an appropriate catalyst were studied, directly on water surface, by grazing incidence X-ray diffraction using synchrotron radiation. The enantiomeric composition of the oligopeptides collected from the interface was determined by matrix-assisted laser-desorption-ionization time-offlight mass spectrometry using deuterium enantio-labeled monomers. Or results show that polymerization within racemic 2-D crystallites can lead to the enhanced generation of oligopeptides with homochiral sequences through reaction between translation-related rather than glide-related molecules. When the reaction occurred primarily between gliderelated molecules, heterochiral oligopeptides were obtained. The latter polymerization was taken advantage of to accomplish an efficient chiral amplification starting with nonracemic mixtures of monomers that undergo segregation into racemic and enantiomorphous crystalline phases. The enantiomer in excess yielded homochiral oligopeptides of single handedness.[1]

Reference:

1. H. Zepik, E. Shavit, M. Tang, T. R. Jensen, K. Kjaer, G. Bolbach, L. Leiserowitz, I. Weissbuch, M. Lahav Science, 259, 1266, (2002) published online 10 January 2002; $10.1126 /$ science. 1065625 .

Keywords: GRAZING INCIDENCE X-RAY DIFFRACTION, SOLID-STATE REACTIONS IN LANGMUIR MONOLAYERS, HOMOCHIRAL OLIGOPEPTIDES

\section{Acta Cryst. (2002). A58 (Supplement), C220}

EPITAXY OF CdS and PbS ON FATTY ACID MONOLAYER: LATTICE MATCHING AND STEREOCHEMICAL RELATIONS E. Rakova N. Stepina V. Klechkovskaya L. Feigin

Affiliations:

Shubnikov Institute of Crystallography

${ }^{1}$ University of Copenhagen Nano-Science Center, Dept. of Chemistry H. C. Orsted Institute Universitetsparken 5 COPENHAGEN 2100 DENMARK

${ }^{2}$ Risoe National Laboratories ${ }^{3}$ Liverpool University

Due to the simple preparation procedure (1) and chemical flexibility ligand stabilized gold nanoparticles have become increasingly important as building blocks for self-assembled nanostructures.

From X-ray diffraction and reflectivity studies of Langmuir films of such systems (2) we have characterized the fundamental assembly process of both hyrdrophobic and chemically responsive amphiphilic nanoparticles on the water surface. It is for example shown how the mobility of ligands allow the clusters to adapt the most favorable structure on the water surface by adjusting the structure of the ligand shell to the particular experimental situation.

The results are related to recent progress of experimental studies of hydrophobic hydration and electronic characterization of gold nanoparticle molecular electronic hybrid structures.

References

(1) M. Brust, M. Walker, D. Bethell, D.J: Schiffrin and R. Whyman, J. Chem. Soc. Chem. Commun., 801 (1994)

(2) M. Brust, N. Stuhr-Hansen, K. Norgaard, J.B. Christensen, L.K. Nielsen and T. Bjornholm, Nano Letters 1, 189-191 (2001)

\section{Keywords: LANGMUIR FILMS GOLD NANO PARTICLES SELF ASSEMBLING ELECTRONICS}

Langmuir monolayers at the air-water interface are used as templates for oriented growth of inorganic nanocrystals. The matter under study is the influence of lattice matching and stereochemical relations on crystal orientation. PbS ( $a=5.94 \AA$, octahedral coordination of atoms)and CdS ( $a=$ $5.83 \AA$, tetrahedral coordination)are grown under behenic acid(BA) monolayer. The structure and mutual orientation are studied by High Energy Electron Diffraction and Transmission Electron Microscopy. The results obtained allow us to conclude that lattice matching of atomic nets at the interface plays the important role in the orientation of $\mathrm{PbS}$ nanocrystals. As opposited to $\mathrm{PbS}$, orientation of CdS nanocrystals corresponds to high stereochemical similarity of organic molecules in 2D-monolayer and CdS-nanocrystals: covalent tetrahedral bonds in CdS and tetrahedral bonds in BA hydrocarbon chains are directed nearly parallel.

Keywords: LANGMUIR MONOLAYER, NANOCRYSTALS, MUTUAL ORIENTATION 